\title{
A family of nonminimizing abnormal curves
}

\section{Roberto Monti}

Received: 7 November 2012 / Accepted: 18 April 2013 / Published online: 27 April 2013

(C) Fondazione Annali di Matematica Pura ed Applicata and Springer-Verlag Berlin Heidelberg 2013

\begin{abstract}
In a four-dimensional sub-Riemannian structure, we study a specific family of abnormal extremals, and we show that they are not length minimizing, answering in the negative to a question that was recently asked. We extend the result to a class of 4-dimensional sub-Riemannian manifolds of step 5.
\end{abstract}

Keywords Sub-Riemannian geometry $\cdot$ Regularity of geodesics $\cdot$ Abnormal extremals

Mathematics Subject Classification $53 \mathrm{C} 17 \cdot 49 \mathrm{~K} 30$

\section{Introduction}

One of the main open problems in sub-Riemannian geometry is the regularity of lengthminimizing curves. Minimizers may be abnormal extremals, in the language of Geometric Control Theory, and R. Montgomery showed in [5] an example of abnormal extremal that is length minimizing. Liu and Sussmann proved later that the existence of length-minimizing abnormal extremals is typical of rank 2 distributions [3].

All known examples of length-minimizing curves are smooth. On the other hand, there is no regularity theory of a general character for sub-Riemannian "geodesics", apart from the partial results of [2] and [6].

During the meeting Geometric control and sub-Riemannian geometry held in Cortona in May 2012, A. Agrachev and J. P. Gauthier suggested the following situation, in order to find a nonsmooth length-minimizing curve.

Consider in $\mathbb{R}^{4}$ the vector fields

$$
X_{1}=\frac{\partial}{\partial x_{1}}+2 x_{2} \frac{\partial}{\partial x_{3}}+x_{3}^{2} \frac{\partial}{\partial x_{4}}, \quad X_{2}=\frac{\partial}{\partial x_{2}}-2 x_{1} \frac{\partial}{\partial x_{3}},
$$

R. Monti (凶)

Padova, Italy

e-mail: monti@math.unipd.it 
and denote by $\mathcal{D}$ the distribution of 2-planes in $\mathbb{R}^{4}$ spanned pointwise by $X_{1}$ and $X_{2}$ :

$$
\mathcal{D}(x)=\operatorname{span}\left\{X_{1}(x), X_{2}(x)\right\}, \quad x \in \mathbb{R}^{4} .
$$

A Lipschitz curve $\gamma:[0,1] \rightarrow \mathbb{R}^{4}$ is horizontal if $\dot{\gamma}(t) \in \mathcal{D}(\gamma(t))$ for a.e. $t \in[0,1]$. Namely, $\gamma$ is horizontal if there exist two functions $h_{1}, h_{1} \in L^{\infty}(0,1)$ such that

$$
\dot{\gamma}=h_{1} X_{1}(\gamma)+h_{2} X_{2}(\gamma) \text { a.e. on }[0,1] \text {. }
$$

The length of $\gamma$ is then defined as

$$
L(\gamma)=\int_{0}^{1} g_{\gamma}(\dot{\gamma}, \dot{\gamma})^{1 / 2} \mathrm{~d} t,
$$

where $g_{x}$ is a metric on $\mathcal{D}(x), x \in \mathbb{R}^{4}$.

Fix a parameter $\alpha>0$ and consider the initial and final points $L=(-1, \alpha, 0,0) \in \mathbb{R}^{4}$ and $R=(1, \alpha, 0,0) \in \mathbb{R}^{4}$. Let $\bar{\gamma}:[-1,1] \rightarrow \mathbb{R}^{4}$ be the curve

$$
\bar{\gamma}_{1}(t)=t, \quad \bar{\gamma}_{2}(t)=\alpha|t|, \quad \bar{\gamma}_{3}(t)=0, \quad \bar{\gamma}_{4}(t)=0, \quad t \in[-1,1] .
$$

The curve $\bar{\gamma}$ is horizontal and joins $L$ to $R$. Moreover, it can be easily checked that $\bar{\gamma}$ is an abnormal extremal in the sense of Geometric Control Theory. The question is whether the curve $\bar{\gamma}$ is length minimizing or not, especially for small $\alpha>0$.

The interest of this question arises from the following consideration. Let $M$ be an $n$ dimensional smooth manifold with $n \geq 3$, and let $\mathcal{D}$ be a completely nonintegrable (i.e., bracket generating) distribution on $M$. Let $\mathcal{D}_{1}=\mathcal{D}$ and $\mathcal{D}_{i}=\left[\mathcal{D}_{1}, \mathcal{D}_{i-1}\right]$, i.e., $\mathcal{D}_{i}$ is the linear span of all commutators $\left[\mathcal{D}_{1}, \mathcal{D}_{i-1}\right]$. We let $\mathcal{L}_{0}=\{0\}$ and $\mathcal{L}_{i}=\mathcal{D}_{1}+\cdots+\mathcal{D}_{i}, i \geq 1$. By the nonintegrability condition, for any $p \in M$, there exists $r \in \mathbb{N}$ such that $\mathcal{L}_{r}(p)=T_{p} M$, the tangent space of $M$ at $p$. Assume that $\mathcal{D}$ is equiregular, i.e., assume that for each $i=1, \ldots, r$

$$
\operatorname{dim}\left(\mathcal{L}_{i}(p) / \mathcal{L}_{i-1}(p)\right) \text { is constant for } p \in M .
$$

In [2], Leonardi and the author proved the following theorem.

Theorem 1.1 Let $(M, \mathcal{D}, g)$ be a sub-Riemannian manifold, where $g$ is a metric on $\mathcal{D}$, that satisfies (1.6) and

$$
\left[\mathcal{L}_{i}, \mathcal{L}_{j}\right] \subset \mathcal{L}_{i+j-1}, \quad i, j \geq 2, i+j>4 .
$$

Then, any curve in $M$ with a corner is not length minimizing in $(M, \mathcal{D}, g)$.

A "curve with a corner" is a $\mathcal{D}$-horizontal curve $\gamma:[0,1] \rightarrow M$ such that at some point $t \in(0,1)$ the left and right derivatives $\dot{\gamma}_{L}(t) \neq \dot{\gamma}_{R}(t)$ exist and are different and nonzero.

In view of Theorem 1.1, it is natural to look for a nonsmooth length-minimizing curve in a sub-Riemannian manifold violating (1.7). The step $r$ associated with $(M, \mathcal{D})$ has to be at least 5, because condition (1.7) is automatically satisfied if $r \leq 4$. A first attempt could be to consider the free Carnot group of step 5 and rank 2. This structure is diffeomorphic to $\mathbb{R}^{8}$ and, by the results in [1], all abnormal extremals are computable.

If we drop the equiregularity condition (1.6), however, the search for structures violating (1.7) is easier. The manifold $M=\mathbb{R}^{4}$ with the distribution $\mathcal{D}$ spanned by the vector fields (1.1) is one such example.

For $n \in \mathbb{N}$, we define the set of multi-indexes $\mathcal{I}_{n}=\{1,2\}^{n}=\left\{\left(\beta_{1}, \ldots, \beta_{n}\right) \in \mathbb{N}^{n}\right.$ : $\left.\beta_{1}, \ldots, \beta_{n}=1,2\right\}$ and, for any $\beta \in \mathcal{I}_{n}$, we let

$$
X_{\beta}=\left[X_{\beta_{1}},\left[\ldots,\left[X_{\beta_{n-1}}, X_{\beta_{n}}\right] \ldots\right]\right] .
$$


We define the length of the commutator $X_{\beta}$ as $\operatorname{len}\left(X_{\beta}\right)=n$ if and only if $\beta \in \mathcal{I}_{n}$.

For any $\beta \in \mathcal{I}_{3} \cup \mathcal{I}_{4}$, we have $X_{\beta}(0)=0$. On the other hand, when $\beta \in \mathcal{I}_{5}$, we have the following situation:

$$
\begin{aligned}
\frac{1}{16} X_{\beta} & =\frac{\partial}{\partial x_{4}}, \quad \text { when } \beta=(1,1,2,2,1) \text { or } \beta=(1,2,1,2,1) \\
\frac{1}{32} X_{\beta} & =-\frac{\partial}{\partial x_{4}}, \quad \text { when } \beta=(2,1,1,2,1) \\
X_{\beta} & =0 \quad \text { otherwise. }
\end{aligned}
$$

In particular, there holds

$$
\frac{1}{48}\left[\left[X_{2}, X_{1}\right],\left[\left[X_{2}, X_{1}\right], X_{1}\right]\right]=\frac{\partial}{\partial x_{4}},
$$

and thus, (1.7) is violated with $i=2$ and $j=3$.

We show that the curve $\bar{\gamma}$ in (1.5) is not length minimizing for any small $\alpha$, thus answering in the negative to the question raised by Agrachev and Gauthier. Namely, we prove the following.

Theorem 1.2 For any $\alpha>0$ with $\alpha \neq 1$, the curve $\bar{\gamma}$ in (1.5) is not length minimizing in $\left(\mathbb{R}^{4}, \mathcal{D}, g\right)$, for any choice of metric $g$ on $\mathcal{D}$.

The proof of the theorem is constructive. For $\alpha \neq 1$, we construct a horizontal curve joining the left and right end-points $L$ and $R$ that is strictly shorter than $\bar{\gamma}$.

When $\alpha=1$, the construction does not work, and our shorter curves for $\alpha \neq 1$ converge to the curve $\bar{\gamma}$ as $\alpha \rightarrow 1$ (see Remark 3.1). The problem of establishing whether $\bar{\gamma}$ for $\alpha=1$ is length minimizing or not is open.

In the second part of the paper, we extend Theorem 1.2 to 4-dimensional sub-Riemannian manifolds having at the corner point of the involved curve the same infinitesimal structure as $\left(\mathbb{R}^{4}, \mathcal{D}\right)$.

Let $M$ be a 4-dimensional smooth manifold, and let $\mathcal{D} \subset T M$ be a distribution of 2-planes on $M$. Locally, we have $\mathcal{D}=\operatorname{span}\left\{Y_{1}, Y_{2}\right\}$, where $Y_{1}$ and $Y_{2}$ are linearly independent smooth vector fields on $M$. We denote by $\mathcal{L}_{2}=\operatorname{span}\left\{Y_{i},\left[Y_{j}, Y_{k}\right]: i, j, k=1,2\right\}$ the pointwise linear span of $\mathcal{D}$ and of the brackets of $\mathcal{D}$.

Fix a point $p \in M$. We make the following three assumptions:

(H1) The vector fields

$$
Y_{1}, Y_{2}, Y_{3}=\frac{1}{4}\left[Y_{2}, Y_{1}\right], \text { and } Y_{4}=\frac{1}{16}\left[Y_{1},\left[Y_{1},\left[Y_{2},\left[Y_{2}, Y_{1}\right]\right]\right]\right]
$$

are linearly independent at the point $p$.

(H2) For all $\beta \in \mathcal{I}_{3} \cup \mathcal{I}_{4}$, we have

$$
Y_{\beta}(p)=0 \bmod \mathcal{L}_{2}(p),
$$

where $\mathcal{L}_{2}(p)=\left\{Y(p): Y \in \mathcal{L}_{2}\right\}$.

(H3) For all $\beta \in \mathcal{I}_{5}$ with $\beta=(*, *, *, 2,1)$, we have

$$
\begin{aligned}
& Y_{\beta}(p)=16 Y_{4}(p) \bmod \mathcal{L}_{2}(p), \text { for } \beta=(1,2,1,2,1) \text { or } \beta=(1,1,2,2,1), \\
& Y_{\beta}(p)=-32 Y_{4}(p) \bmod \mathcal{L}_{2}(p), \text { for } \beta=(2,1,1,2,1), \text { and } \\
& Y_{\beta}(p)=0 \bmod \mathcal{L}_{2}(p) \text { otherwise. }
\end{aligned}
$$


Finally, let $g$ be a metric on $\mathcal{D}$ making the vector fields $Y_{1}$ and $Y_{2}$ orthogonal at $p$,

$$
g_{p}\left(Y_{1}, Y_{2}\right)=0 .
$$

In Sect. 5, we prove the following result. Below, we denote by $\dot{\gamma}_{L}(t)$ and $\dot{\gamma}_{R}(t)$ the left and right derivative of a curve $\gamma:[0,1] \rightarrow M$ at the point $t \in(0,1)$. The curve $\gamma$ has a corner at the point $p=\gamma(t)$, if the left and right derivatives at $t$ exist, do not vanish, and $\dot{\gamma}_{L}(t) \neq \dot{\gamma}_{R}(t)$.

Theorem 1.3 Let $(M, \mathcal{D}, g)$ be 4-dimensional sub-Riemannian manifold satisfying (H1)(H3) and (1.13) at the point $p \in M$. Let $\gamma:[0,1] \rightarrow M$ be a horizontal curve in $(M, \mathcal{D})$ having a corner at the point $p=\gamma(t), t \in(0,1)$, such that $g_{p}\left(\dot{\gamma}_{L}(t), \dot{\gamma}_{R}(t)\right) \neq 0$. Then, $\gamma$ is not length minimizing.

The proof of Theorem 1.3 relies upon a blow-up argument reducing the situation to the one of the Theorem 1.2. Theorem 1.3 is proved in Sect. 5, and the preliminary nilpotent approximation is explained in Sect. 4. In Sect. 2, we set up the construction of the shorter curve used to prove Theorem 1.2. In Sect. 3, we solve a system of end-point equations, concluding the proof of Theorem 1.2.

We thank D. Vittone for his careful reading of an early version of this paper. We also thank the referee for suggesting to us possible extensions of Theorem 1.2.

\section{Construction of the competing curve}

In this section, we construct the competing curve used to prove Theorem 1.2. In a first step, we cut the corner of $\bar{\gamma}$ at $t=0$ in the $x_{1} x_{2}$-plane. Lifting the new curve in $\mathbb{R}^{2}$ to a horizontal curve in $\mathbb{R}^{4}$ produces an error on the third and fourth coordinates of the final point. To restore the final point, we use two devices. We describe the intuition behind the construction. The vector fields $X_{1}$ and $X_{2}$ in (1.1) have a Heisenberg group structure in the $x_{1} x_{2} x_{3}$ space. Thus, to restore the third coordinate, we have to add an "area" in the $x_{1} x_{2}$-plane equaling the "area" cut at the corner. We do this by means of a "rectangle" having basis of fixed length and variable height $\varepsilon_{1}$, the first parameter.

Restoring the fourth coordinate is more delicate. This is due to the fact that the coefficient of $\partial / \partial x_{4}$ of $X_{1}$ in (1.1), the power $x_{3}^{2}$, is always nonnegative. This means that the cut produces a positive error, see (2.7). To correct it, the perturbation of $\bar{\gamma}$ must contain arcs where the first coordinate is decreasing. To do this, we add a square with side length $\varepsilon_{2}$, the second parameter, at the final point $R$ in the $x_{1} x_{2}$ plane.

Our goal is to prove that the final end-point can be adjusted via this construction. Technically, this means that we have to find solutions $\varepsilon_{1}, \varepsilon_{2}$ to a system of two equations depending on the cut parameter. Moreover, we need to check that the cost of length of "rectangle" and square does not exceed the gain of length of the cut. This will be done in Sect. 3.

We begin the construction of the shorter curve.

\subsection{Cut}

Fix a cut parameter $0<\eta<1 / 4$, and let

$$
T_{\eta}=\left\{\left(x_{1}, x_{2}\right) \in \mathbb{R}^{2}: \alpha\left|x_{1}\right|<x_{2}<\alpha \eta\right\} .
$$

The curve $\bar{\gamma}$ is deviated along the cut, i.e., along the side of the triangle $T_{\eta}$ parallel to the $x_{1}$-axis. In other words, we cut the corner. The cut produces an error on the final point, namely 
on the third and fourth coordinates. To correct these errors, we use two devices, a "rectangle" and a square.

\subsection{Rectangle}

We deviate $\bar{\gamma}$ along a "rectangle" put along $\bar{\gamma}$. The length of the basis is fixed. The height is a variable parameter. Namely, for $\varepsilon_{1}>0$, we let

$$
R_{\varepsilon_{1}}=\left\{\left(x_{1}, x_{2}\right) \in \mathbb{R}^{2}: \frac{1}{4}<x_{1}<\frac{1}{2}, \alpha x_{1}<x_{2}<\alpha x_{1}+\varepsilon_{1}\right\} .
$$

The curve $\bar{\gamma}$ is deviated following clockwise three sides of the rectangle. When $\varepsilon_{1}<0$, the construction is analogous, but the rectangle is below the curve, and we follow its boundary counterclockwise.

\subsection{Square}

Next, we use a square with bottom-left vertex at $R$, the final point. Namely, for any $\varepsilon_{2} \in \mathbb{R}$, we let:

$$
Q_{\varepsilon_{2}}=\left\{\left(x_{1}, x_{2}\right) \in \mathbb{R}^{2}: 1<x_{1}<1+\left|\varepsilon_{2}\right|, \alpha<x_{2}<\alpha+\left|\varepsilon_{2}\right|\right\} .
$$

When $\varepsilon_{2}>0$, we follow the boundary of the square clockwise. When $\varepsilon_{2}<0$, we follow the boundary counterclockwise.

The devices $R_{\varepsilon_{1}}$ and $Q_{\varepsilon_{2}}$ produce effects in the coordinates 3 and 4 of $\bar{\gamma}$ after the cut. We call $\gamma:[-1,1] \rightarrow \mathbb{R}^{4}$ the curve obtained after using $T_{\eta}, R_{\varepsilon_{1}}$, and $Q_{\varepsilon_{2}}$. To construct the coordinate $\gamma_{3}$, we use the formula

$$
\gamma_{3}(t)=2 \int_{-1}^{t}\left(\gamma_{2} \dot{\gamma}_{1}-\gamma_{1} \dot{\gamma}_{2}\right) \mathrm{d} s, \quad t \in[0,1] .
$$

To construct $\gamma_{4}$, we use the formula

$$
\gamma_{4}(t)=\int_{-1}^{t} \dot{\gamma}_{1} \gamma_{3}^{2} \mathrm{~d} s, \quad t \in[0,1] .
$$

On suitable subintervals, we shall use different parameterizations for $\gamma$.

We construct step by step the horizontal lift of $\gamma$.

\subsection{Effect of $T_{\eta}$}

We parameterize on the interval $[-\eta, \eta]$ the piece of $\gamma$ that is on the horizontal part of $\partial T_{\eta}$. We have, by (2.1),

$$
\gamma_{3}(t)=\int_{-\eta}^{t} 2 \alpha \eta \mathrm{d} s=2 \alpha \eta(t+\eta), \quad t \in[-\eta, \eta] .
$$

The final value of $\gamma_{3}$ is $\gamma_{3}(\eta)=4 \alpha \eta^{2}$. The final value of $\gamma_{4}$ is, by (2.2),

$$
\gamma_{4}(\eta)=\int_{-\eta}^{\eta} 4 \alpha^{2} \eta^{2}(t+\eta)^{2} \mathrm{~d} t=\frac{32}{3} \alpha^{2} \eta^{5} .
$$




\subsection{First segment}

Now, we have to follow for a time $t \in[\eta, 1 / 4]$ the piece of the original curve $\bar{\gamma}$ connecting the cut to the rectangle $R_{\varepsilon_{1}}$. Here, $\gamma_{3}$ is constant: $\gamma_{3} \equiv 4 \alpha \eta^{2}$. The final value of $\gamma_{4}$ is

$$
\gamma_{4}(1 / 4)=\frac{32}{3} \alpha^{2} \eta^{5}+\int_{\eta}^{1 / 4} 16 \alpha^{2} \eta^{4} \mathrm{~d} t=16 \alpha^{2} \eta^{4}\left(\frac{1}{4}-\frac{\eta}{3}\right) .
$$

\subsection{Rectangle}

$\mathbf{1}^{\text {st }}$ side. We compute $\gamma_{3}$ along the first vertical side $\left\{(1 / 4, \alpha / 4+t) \in \mathbb{R}^{2}: t \in\left[0, \varepsilon_{1}\right]\right\}$ of $R_{\varepsilon_{1}}$ :

$$
\gamma_{3}(t)=4 \alpha \eta^{2}-\frac{t}{2}, \quad t \in\left[0, \varepsilon_{1}\right]
$$

The final value is $\gamma_{3}\left(\varepsilon_{1}\right)=4 \alpha \eta^{2}-\frac{\varepsilon_{1}}{2}$. This value is correct also when $\varepsilon_{1}<0$. Along this piece of curve, $\gamma_{4}$ stays constant because $\dot{\gamma}_{1}=0$.

$2^{\text {nd }}$ side. Along the second side of the rectangle, we have

$$
\gamma_{1}(t)=\frac{1}{4}+t, \quad \gamma_{2}(t)=\frac{\alpha}{4}+\varepsilon_{1}+\alpha t, \quad t \in[0,1 / 4] .
$$

Then, the third coordinate is

$$
\begin{aligned}
\gamma_{3}(t) & =4 \alpha \eta^{2}-\frac{\varepsilon_{1}}{2}+2 \varepsilon_{1} t, \quad t \in[0,1 / 4], \\
\gamma_{3}(1 / 4) & =4 \alpha \eta^{2} .
\end{aligned}
$$

The final value of $\gamma_{4}$ at the end of this side is

$$
\begin{aligned}
\gamma_{4}(1 / 4) & =16 \alpha^{2} \eta^{4}\left(\frac{1}{4}-\frac{\eta}{3}\right)+\int_{0}^{1 / 4}\left(4 \alpha \eta^{2}-\frac{\varepsilon_{1}}{2}+2 \varepsilon_{1} t\right)^{2} \mathrm{~d} t \\
& =16 \alpha^{2} \eta^{4}\left(\frac{1}{4}-\frac{\eta}{3}\right)+4 \alpha^{2} \eta^{4}-\frac{1}{2} \alpha \eta^{2} \varepsilon_{1}+\frac{1}{48} \varepsilon_{1}^{2} \\
& =\varrho
\end{aligned}
$$

This is the final value of $\gamma_{4}$ after the rectangle $R_{\varepsilon_{1}}$. The number $\varrho$ is defined via the last identity in (2.3).

$3^{\text {rd }}$ side. We compute $\gamma_{3}$ along the second vertical side $\left\{(1 / 2, \alpha / 2+t) \in \mathbb{R}^{2}: t \in\left[0, \varepsilon_{1}\right]\right\}$ of the rectangle $R_{\varepsilon_{1}}$. We have to follow $-X_{2}$, and we get

$$
\begin{aligned}
\gamma_{3}(t) & =4 \alpha \eta^{2}+t, \quad t \in\left[0, \varepsilon_{1}\right], \\
\gamma_{3}\left(\varepsilon_{1}\right) & =4 \alpha \eta^{2}+\varepsilon_{1} .
\end{aligned}
$$

Here, $\gamma_{4}$ is constant because $\dot{\gamma}_{1}=0$ on this side of the rectangle.

2.7 Second diagonal segment

Now, we have to follow for a time $t \in[1 / 2,1]$ the piece of the original curve $\bar{\gamma}$ connecting $R_{\varepsilon_{1}}$ to the final point $R$. Here, $\gamma_{3}$ is constant: $\gamma_{3} \equiv 4 \alpha \eta^{2}+\varepsilon_{1}$. We call $\Delta_{0}\left(\varepsilon_{1} ; \eta\right)$ the final 
value of $\gamma_{4}$ before the square $Q_{\varepsilon_{2}}$, and namely [recall the definition of $\varrho$ in (2.3)],

$$
\begin{aligned}
\Delta_{0}\left(\varepsilon_{1} ; \eta\right) & =\varrho+\frac{1}{2}\left(4 \alpha \eta^{2}+\varepsilon_{1}\right)^{2} \\
& =16 \alpha^{2} \eta^{4}\left(1-\frac{\eta}{3}\right)+\frac{7}{2} \alpha \eta^{2} \varepsilon_{1}+\frac{25}{48} \varepsilon_{1}^{2} .
\end{aligned}
$$

\subsection{Square}

Case $\varepsilon_{2}>0$. We compute the effect on the third and fourth coordinates of the square $Q_{\varepsilon_{2}}$. Here, we do the computations for the case $\varepsilon_{2}>0$. In this case, we follow the boundary $\partial Q_{\varepsilon_{2}}$ clockwise.

$\mathbf{1}^{\text {st }}$ vertical side. We follow the segment $\left\{(1, \alpha+t) \in \mathbb{R}^{2}: t \in\left[0, \varepsilon_{2}\right]\right\}$. The coordinate $\gamma_{3}$ is

$$
\begin{aligned}
\gamma_{3}(t) & =4 \alpha \eta^{2}+\varepsilon_{1}-2 t, \quad t \in\left[0, \varepsilon_{2}\right], \\
\gamma_{3}\left(\varepsilon_{2}\right) & =4 \alpha \eta^{2}+\varepsilon_{1}-2 \varepsilon_{2} .
\end{aligned}
$$

The coordinate $\gamma_{4}$ stays constant along this side, $\gamma_{4} \equiv \Delta_{0}\left(\varepsilon_{1} ; \eta\right)$.

$2^{\text {nd }}$ (horizontal) side. We follow the line segment $\left\{\left(1+t, \alpha+\varepsilon_{2}\right) \in \mathbb{R}^{2}: t \in\left[0, \varepsilon_{2}\right]\right\}$. The coordinate $\gamma_{3}$ is

$$
\begin{aligned}
\gamma_{3}(t) & =4 \alpha \eta^{2}+\varepsilon_{1}-2 \varepsilon_{2}+2\left(\alpha+\varepsilon_{2}\right) t, \quad t \in\left[0, \varepsilon_{2}\right] \\
\gamma_{3}\left(\varepsilon_{2}\right) & =4 \alpha \eta^{2}+\varepsilon_{1}-2 \varepsilon_{2}+2\left(\alpha+\varepsilon_{2}\right) \varepsilon_{2} .
\end{aligned}
$$

Here, there is an important contribution to the fourth coordinate:

$$
\begin{aligned}
\gamma_{4}\left(\varepsilon_{2}\right)= & \Delta_{0}\left(\varepsilon_{1} ; \eta\right)+\int_{0}^{\varepsilon_{2}}\left(4 \alpha \eta^{2}+\varepsilon_{1}-2 \varepsilon_{2}+2\left(\alpha+\varepsilon_{2}\right) t\right)^{2} \mathrm{~d} t \\
= & \Delta_{0}\left(\varepsilon_{1} ; \eta\right)+\left(4 \alpha \eta^{2}+\varepsilon_{1}-2 \varepsilon_{2}\right)^{2} \varepsilon_{2} \\
& +2\left(4 \alpha \eta^{2}+\varepsilon_{1}-2 \varepsilon_{2}\right)\left(\alpha+\varepsilon_{2}\right) \varepsilon_{2}^{2}+\frac{4}{3}\left(\alpha+\varepsilon_{2}\right)^{2} \varepsilon_{2}^{3} \\
= & \Delta_{1}\left(\varepsilon_{2}, \varepsilon_{2} ; \eta\right) .
\end{aligned}
$$

Above, $\Delta_{1}\left(\varepsilon_{2}, \varepsilon_{2} ; \eta\right)$ is defined via the last identity.

$\mathbf{3}^{\text {rd }}$ (vertical) side. We have to follow the vertical side $\left\{\left(1+\varepsilon_{2}, \alpha+t\right) \in \mathbb{R}^{2}: t \in\left[0, \varepsilon_{2}\right]\right\}$ along the vector field $-X_{2}$. The final coordinate $\gamma_{3}$ is:

$$
\gamma_{3}\left(\varepsilon_{2}\right)=4 \alpha \eta^{2}+\varepsilon_{1}+2 \alpha \varepsilon_{2}+4 \varepsilon_{2}^{2} .
$$

The coordinate $\gamma_{4}$ does not change.

$4^{\text {th }}$ (horizontal) side. We have to follow the horizontal side $\left\{(1+t, \alpha) \in \mathbb{R}^{2}: t \in\left[0, \varepsilon_{2}\right]\right\}$ along the vector field $-X_{1}$. The coordinate $\gamma_{3}$ is as follows:

$$
\begin{aligned}
\gamma_{3}(t) & =4 \alpha \eta^{2}+\varepsilon_{1}+2 \alpha \varepsilon_{2}+4 \varepsilon_{2}^{2}-2 \alpha t, \quad t \in\left[0, \varepsilon_{2}\right] \\
\gamma_{3}\left(\varepsilon_{2}\right) & =4 \alpha \eta^{2}+\varepsilon_{1}+4 \varepsilon_{2}^{2} .
\end{aligned}
$$

This is the final value of $\gamma_{3}$ after the entire construction, when $\varepsilon_{2}>0$. When $\varepsilon_{2}<0$, we have to replace $\varepsilon_{2}^{2}$ above with $\operatorname{sgn}\left(\varepsilon_{2}\right)\left|\varepsilon_{2}\right|^{2}$. There is a change of sign. We call $\Gamma_{3}\left(\varepsilon_{1}, \varepsilon_{2} ; \eta\right)$ the final value of $\gamma_{3}$ after the entire construction, and namely,

$$
\Gamma_{3}\left(\varepsilon_{1}, \varepsilon_{2} ; \eta\right)=4 \alpha \eta^{2}+\varepsilon_{1}+4 \operatorname{sgn}\left(\varepsilon_{2}\right)\left|\varepsilon_{2}\right|^{2} .
$$


The final value of $\gamma_{4}$ is as follows:

$$
\begin{aligned}
\gamma_{4}\left(\varepsilon_{2}\right)= & \Delta_{1}\left(\varepsilon_{2}, \varepsilon_{2} ; \eta\right)-\int_{0}^{\varepsilon_{2}}\left(4 \alpha \eta^{2}+\varepsilon_{1}+2 \alpha \varepsilon_{2}+4 \varepsilon_{2}^{2}-2 \alpha t\right)^{2} \mathrm{~d} t \\
= & \Delta_{1}\left(\varepsilon_{2}, \varepsilon_{2} ; \eta\right)-\left[\left(4 \alpha \eta^{2}+\varepsilon_{1}+4 \varepsilon_{2}^{2}\right)^{2} \varepsilon_{2}+2\left(4 \alpha \eta^{2}+\varepsilon_{1}+4 \varepsilon_{2}^{2}\right) \alpha \varepsilon_{2}^{2}+\frac{4}{3} \alpha^{2} \varepsilon_{2}^{3}\right] \\
= & \Delta_{0}\left(\varepsilon_{1} ; \eta\right)+4 \varepsilon_{2}^{2}\left(1+2 \varepsilon_{2}\right)\left[\varepsilon_{2}\left(1-2 \varepsilon_{2}\right)-4 \alpha \eta^{2}-\varepsilon_{1}\right] \\
& +2 \varepsilon_{2}^{3}\left[4 \alpha \eta^{2}+\varepsilon_{1}-2\left(\alpha+\varepsilon_{2}\right)-4 \alpha \varepsilon_{2}\right]+\frac{4}{3} \varepsilon_{2}^{4}\left(2 \alpha+\varepsilon_{2}\right) .
\end{aligned}
$$

Recalling (2.4), we let for $\varepsilon_{2}>0$

$$
\begin{aligned}
\Gamma_{4}\left(\varepsilon_{1}, \varepsilon_{2} ; \eta\right)= & 16 \alpha^{2} \eta^{4}\left(1-\frac{\eta}{3}\right)+\frac{7}{2} \alpha \eta^{2} \varepsilon_{1}+\frac{25}{48} \varepsilon_{1}^{2} \\
& +4 \varepsilon_{2}^{2}\left(1+2 \varepsilon_{2}\right)\left[\varepsilon_{2}\left(1-2 \varepsilon_{2}\right)-4 \alpha \eta^{2}-\varepsilon_{1}\right] \\
& +2 \varepsilon_{2}^{3}\left[4 \alpha \eta^{2}+\varepsilon_{1}-2\left(\alpha+\varepsilon_{2}\right)-4 \alpha \varepsilon_{2}\right]+\frac{4}{3} \varepsilon_{2}^{4}\left(2 \alpha+\varepsilon_{2}\right) .
\end{aligned}
$$

\subsection{Square}

Case $\varepsilon_{2}<0$. We compute the effect of the square $Q_{\varepsilon_{2}}$ on the fourth coordinate when $\varepsilon_{2}<0$. In this case, we follow $\partial Q_{\varepsilon_{2}}$ counterclockwise.

$\mathbf{1}^{\text {st }}$ horizontal side. We follow the segment $\left\{(1+t, \alpha) \in \mathbb{R}^{2}: t \in\left[0,\left|\varepsilon_{2}\right|\right]\right\}$. The coordinate $\gamma_{3}$ is

$$
\begin{aligned}
\gamma_{3}(t) & =4 \alpha \eta^{2}+\varepsilon_{1}+2 \alpha t, \quad t \in\left[0,\left|\varepsilon_{2}\right|\right], \\
\gamma_{3}\left(\left|\varepsilon_{2}\right|\right) & =4 \alpha \eta^{2}+\varepsilon_{1}+2 \alpha\left|\varepsilon_{2}\right| .
\end{aligned}
$$

The final value of the coordinate $\gamma_{4}$ is

$$
\begin{aligned}
\gamma_{4}\left(\left|\varepsilon_{2}\right|\right) & =\Delta_{0}\left(\varepsilon_{1} ; \eta\right)+\int_{0}^{\left|\varepsilon_{2}\right|}\left(4 \alpha \eta^{2}+\varepsilon_{1}+2 \alpha t\right)^{2} \mathrm{~d} t \\
& =\Delta_{0}\left(\varepsilon_{1} ; \eta\right)+\left|\varepsilon_{2}\right|\left(4 \alpha \eta^{2}+\varepsilon_{1}\right)^{2}+2 \alpha\left|\varepsilon_{2}\right|^{2}\left(4 \alpha \eta^{2}+\varepsilon_{1}\right)+\frac{4}{3} \alpha^{2}\left|\varepsilon_{2}\right|^{3} \\
& =\Delta_{1}\left(\varepsilon_{1}, \varepsilon_{2} ; \eta\right) .
\end{aligned}
$$

Here, $\Delta_{1}\left(\varepsilon_{1}, \varepsilon_{2}\right)$ for $\varepsilon_{2}<0$ is defined via the last identity.

$2^{\text {nd }}$ (vertical) side. We follow the line segment $\left\{\left(1+\left|\varepsilon_{2}\right|, \alpha+t\right) \in \mathbb{R}^{2}: t \in\left[0,\left|\varepsilon_{2}\right|\right]\right\}$. The coordinate $\gamma_{3}$ is

$$
\begin{aligned}
\gamma_{3}(t) & =4 \alpha \eta^{2}+\varepsilon_{1}+2 \alpha\left|\varepsilon_{2}\right|-2\left(1+\left|\varepsilon_{2}\right|\right) t, \quad t \in\left[0,\left|\varepsilon_{2}\right|\right] \\
\gamma_{3}\left(\left|\varepsilon_{2}\right|\right) & =4 \alpha \eta^{2}+\varepsilon_{1}+2 \alpha\left|\varepsilon_{2}\right|-2\left(1+\left|\varepsilon_{2}\right|\right)\left|\varepsilon_{2}\right| .
\end{aligned}
$$

The fourth coordinate does not change.

$3^{\text {rd }}$ (horizontal) side. We have to follow the horizontal side $\left\{\left(1+t, \alpha+\left|\varepsilon_{2}\right|\right) \in \mathbb{R}^{2}: t \in\right.$ $\left.\left[0,\left|\varepsilon_{2}\right|\right]\right\}$ along the vector field $-X_{1}$. The coordinate $\gamma_{3}$ is:

$$
\begin{gathered}
\gamma_{3}(t)=4 \alpha \eta^{2}+\varepsilon_{1}+2 \alpha\left|\varepsilon_{2}\right|-2\left(1+\left|\varepsilon_{2}\right|\right)\left|\varepsilon_{2}\right|-2\left(\alpha+\left|\varepsilon_{2}\right|\right) t, \\
\gamma_{3}\left(\left|\varepsilon_{2}\right|\right)=4 \alpha \eta^{2}+\varepsilon_{1}+2 \alpha\left|\varepsilon_{2}\right|-2\left(1+\left|\varepsilon_{2}\right|\right)\left|\varepsilon_{2}\right|-2\left(\alpha+\left|\varepsilon_{2}\right|\right)\left|\varepsilon_{2}\right|,
\end{gathered}
$$


The final value of the coordinate $\gamma_{4}$ is

$$
\begin{aligned}
\gamma_{4}\left(\left|\varepsilon_{2}\right|\right)= & \Delta_{1}\left(\varepsilon_{1}, \varepsilon_{2} ; \eta\right)-\int_{0}^{\left|\varepsilon_{2}\right|}\left(4 \alpha \eta^{2}+\varepsilon_{1}+2 \alpha\left|\varepsilon_{2}\right|-2\left(1+\left|\varepsilon_{2}\right|\right)\left|\varepsilon_{2}\right|-2\left(\alpha+\left|\varepsilon_{2}\right|\right) t\right)^{2} \mathrm{~d} t \\
= & \Delta_{1}\left(\varepsilon_{1}, \varepsilon_{2} ; \eta\right)-\left|\varepsilon_{2}\right|\left(4 \alpha \eta^{2}+\varepsilon_{1}+2 \alpha\left|\varepsilon_{2}\right|-2\left(1+\left|\varepsilon_{2}\right|\right)\left|\varepsilon_{2}\right|\right)^{2} \\
& +2\left(\alpha+\left|\varepsilon_{2}\right|\right)\left|\varepsilon_{2}\right|^{2}\left(4 \alpha \eta^{2}+\varepsilon_{1}+2 \alpha\left|\varepsilon_{2}\right|-2\left(1+\left|\varepsilon_{2}\right|\right)\left|\varepsilon_{2}\right|\right)-\frac{4}{3}\left(\alpha+\left|\varepsilon_{2}\right|\right)^{2}\left|\varepsilon_{2}\right|^{3} .
\end{aligned}
$$

This is the final value of $\gamma_{4}$ after the entire construction when $\varepsilon_{2}<0$. Then, for $\varepsilon_{2}<0$ we let

$$
\begin{aligned}
\Gamma_{4}\left(\varepsilon_{1}, \varepsilon_{2} ; \eta\right)= & 16 \alpha^{2} \eta^{4}\left(1-\frac{\eta}{3}\right)+\frac{7}{2} \alpha \eta^{2} \varepsilon_{1}+\frac{25}{48} \varepsilon_{1}^{2} \\
& +\left|\varepsilon_{2}\right|\left(4 \alpha \eta^{2}+\varepsilon_{1}\right)^{2}+2 \alpha\left|\varepsilon_{2}\right|^{2}\left(4 \alpha \eta^{2}+\varepsilon_{1}\right)+\frac{4}{3} \alpha^{2}\left|\varepsilon_{2}\right|^{3} \\
& -\left|\varepsilon_{2}\right|\left(4 \alpha \eta^{2}+\varepsilon_{1}+2 \alpha\left|\varepsilon_{2}\right|-2\left(1+\left|\varepsilon_{2}\right|\right)\left|\varepsilon_{2}\right|\right)^{2} \\
& +2\left(\alpha+\left|\varepsilon_{2}\right|\right)\left|\varepsilon_{2}\right|^{2}\left(4 \alpha \eta^{2}+\varepsilon_{1}+2 \alpha\left|\varepsilon_{2}\right|-2\left(1+\left|\varepsilon_{2}\right|\right)\left|\varepsilon_{2}\right|\right) \\
& -\frac{4}{3}\left(\alpha+\left|\varepsilon_{2}\right|\right)^{2}\left|\varepsilon_{2}\right|^{3} .
\end{aligned}
$$

\section{Proof of Theorem 1.2}

In this section, we complete the proof of Theorem 1.2. We show that the system of end-point equations has a solution, and we prove that, for a small $\eta>0$, this solution provides a curve that is shorter than $\bar{\gamma}$.

To correct the coordinates $\gamma_{3}$ and $\gamma_{4}$, we have to find solutions $\varepsilon_{1}$ and $\varepsilon_{2}$, depending on the (small) parameter $\eta>0$, to the following system of equations

$$
\left\{\begin{array}{l}
\Gamma_{3}\left(\varepsilon_{1}, \varepsilon_{2} ; \eta\right)=0 \\
\Gamma_{4}\left(\varepsilon_{1}, \varepsilon_{2} ; \eta\right)=0
\end{array}\right.
$$

Here, $\Gamma_{4}\left(\varepsilon_{1}, \varepsilon_{2} ; \eta\right)$ is defined in (2.6) and (2.7), for $\varepsilon_{2}>0$ and $\varepsilon_{2}<0$, respectively.

By $(2.5)$, the first equation $\Gamma_{3}\left(\varepsilon_{1}, \varepsilon_{2} ; \eta\right)=0$ is

$$
4 \alpha \eta^{2}+\varepsilon_{1}+4 \operatorname{sgn}\left(\varepsilon_{2}\right)\left|\varepsilon_{2}\right|^{2}=0
$$

We compute $\varepsilon_{1}$ using Eq. (3.2) and replace this value into the second equation $\Gamma_{4}\left(\varepsilon_{1}, \varepsilon_{2} ; \eta\right)=0$.

\subsection{Case $\alpha>1$}

In this case, we look for a solution $\varepsilon_{2}>0$, and we use formula (2.6) for $\Gamma_{4}\left(\varepsilon_{1}, \varepsilon_{2} ; \eta\right)$. Letting $\varepsilon_{2}=\sigma>0$, we obtain the following equation in $\sigma$

$$
\begin{aligned}
& \frac{1}{3}\left(31 \alpha^{2} \eta^{4}-16 \alpha^{2} \eta^{5}+8 \alpha \eta^{2} \sigma^{2}+25 \sigma^{4}\right) \\
& \quad+4 \sigma^{3}\left(1-\alpha+\sigma(3-2 \alpha)+2 \sigma^{2}\right)+\frac{4}{3} \sigma^{4}(2 \alpha+\sigma)=0 .
\end{aligned}
$$


When $\alpha>1$ Eq. (3.3) reads

$$
\phi(\sigma)=\alpha^{2} \eta^{4}(31-16 \eta)+8 \alpha \eta^{2} \sigma^{2}+12(1-\alpha) \sigma^{3}+o\left(\sigma^{3}\right)=0,
$$

where $o\left(\sigma^{3}\right)$ contains terms with $\sigma^{4}$ and $\sigma^{5}$. Notice that the coefficient of $\sigma^{3}$ is negative, because $\alpha>1$. The function $\phi$ introduced in (3.4) is continuous, and moreover,

$$
\phi(0)=\alpha^{2} \eta^{4}(31-16 \eta)>0,
$$

as soon as $\eta<31 / 16$. In fact, we have $\eta<1 / 4$. On the other hand, we have

$$
\phi\left(C \sqrt[3]{\frac{\alpha^{2}}{\alpha-1}} \eta^{4 / 3}\right)=\alpha^{2} \eta^{4}\left(31-12 C^{3}\right)+o\left(\eta^{4}\right)<0,
$$

provided that we choose a constant $C$ such that $12 C^{3}>31$ and $\eta>0$ is small enough.

By the theorem of zeros for continuous functions, for any $\eta>0$ that is small enough, Eq. (3.4) has a solution $\varepsilon_{2}=\sigma>0$ such that

$$
\varepsilon_{2}=\sigma \leq C \sqrt[3]{\frac{\alpha^{2}}{\alpha-1}} \eta^{4 / 3} .
$$

Moreover, by (3.2),

$$
\varepsilon_{1}=-4 \alpha \eta^{2}-4 \operatorname{sgn}\left(\varepsilon_{2}\right)\left|\varepsilon_{2}\right|^{2}=-4 \alpha \eta^{2}-4 \varepsilon_{2}^{2} .
$$

In particular, $\varepsilon_{1}$ is asymptotic to $-4 \alpha \eta^{2}$, for small $\eta>0$, and thus, we have

$$
\left|\varepsilon_{1}\right| \leq 5 \alpha \eta^{2}
$$

for all $\eta>0$ that are sufficiently small.

\subsection{Case $0<\alpha<1$}

In this case, we look for solutions $\varepsilon_{1}, \varepsilon_{2}$ to the system (3.1) such that $\varepsilon_{2}<0$. The unknown $\varepsilon_{1}$ is determined by (3.2). We use formula (2.7) for $\Gamma_{4}\left(\varepsilon_{1}, \varepsilon_{2} ; \eta\right)$. Letting $\sigma=\left|\varepsilon_{2}\right|>0$, equation $\Gamma_{4}\left(\varepsilon_{1}, \varepsilon_{2} ; \eta\right)=0$ reads

$$
\begin{aligned}
& 16 \alpha^{2} \eta^{4}\left(1-\frac{\eta}{3}\right)+14 \alpha \eta^{2}\left(\sigma^{2}-\alpha \eta^{2}\right)+\frac{25}{3}\left(\sigma^{2}-\alpha \eta^{2}\right)^{2}+16 \sigma^{5}+8 \alpha \sigma^{4} \\
& +\frac{4}{3} \alpha^{2} \sigma^{3}-4 \sigma^{3}(\sigma+\alpha-1)^{2}+4(\alpha+\sigma) \sigma^{3}(\sigma+\alpha-1)-\frac{4}{3}(\alpha+\sigma)^{2} \sigma^{3}=0 .
\end{aligned}
$$

We may shorten Eq. (3.7) in the following way:

$$
\alpha^{2} \eta^{4}(31-16 \eta)-8 \alpha \eta^{2} \sigma^{2}+12(\alpha-1) \sigma^{3}+o\left(\sigma^{3}\right)=0 .
$$

Compare Eqs. (3.8) and (3.4). Notice that the coefficient of $\sigma^{3}$ in (3.8) is negative. The same argument used in the case $\alpha>1$ proves that there exists a solution $\left|\varepsilon_{2}\right|=\sigma$ to Eq. (3.8) such that

$$
0<\left|\varepsilon_{2}\right|=\sigma \leq C \sqrt[3]{\frac{\alpha^{2}}{1-\alpha}} \eta^{4 / 3},
$$


where $C$ is the same constant as in the case $\alpha>1$. As above, we deduce that

$$
\left|\varepsilon_{1}\right| \leq 5 \alpha \eta^{2} \text {. }
$$

\subsection{Gain of length}

We compute the difference between the length of the original curve $\bar{\gamma}$ and the curve $\gamma$ obtained after applying the cut $T_{\eta}$, the "rectangle" $R_{\varepsilon_{1}}$, and the square $Q_{\varepsilon_{2}}$

Without loss of generality, we can assume that the metric $g$ on $\mathcal{D}$ is the one making $X_{1}$ and $X_{1}$ orthonormal. In this case, the length $L(\gamma)$ of a horizontal curve $\gamma$ as in (1.4) is

$$
L(\gamma)=\int_{0}^{1}|h(t)| \mathrm{d} t, \quad h=\left(h_{1}, h_{2}\right) .
$$

By (3.11), the gain of length $G(\eta)$ obtained through the cut $T_{\eta}$ is

$$
G(\eta)=2 \eta\left(\sqrt{1+\alpha^{2}}-1\right) .
$$

By (3.6) and (3.9), the cost of length $C_{R}\left(\varepsilon_{1}\right)$ of the correction made through the "rectangle" $R_{\varepsilon_{1}}$ is

$$
C_{R}\left(\varepsilon_{1}\right)=2\left|\varepsilon_{1}\right| \leq 10 \alpha \eta^{2} .
$$

By (3.5) and (3.9), the cost of length $C_{Q}\left(\varepsilon_{2}\right)$ of the correction made through the square $Q_{\varepsilon_{2}}$ is

$$
C_{Q}\left(\varepsilon_{2}\right)=4\left|\varepsilon_{2}\right| \leq 4 C \sqrt[3]{\frac{\alpha^{2}}{\alpha-1}} \eta^{4 / 3}
$$

We conclude that

$$
G(\eta)-C_{R}\left(\varepsilon_{1}\right)-C_{Q}\left(\varepsilon_{2}\right) \geq 2 \eta\left(\sqrt{1+\alpha^{2}}-1\right)-10 \alpha \eta^{2}-4 C \sqrt[3]{\frac{\alpha^{2}}{\alpha-1}} \eta^{4 / 3}>0
$$

for all $\eta>0$ that are sufficiently small.

Thus, the construction provides a horizontal curve $\gamma$ shorter than $\bar{\gamma}$ and joining the same end-points $L$ and $R$. This ends the proof of Theorem 1.2.

Remark 3.1 When $\alpha \rightarrow 1$, our solutions $\varepsilon_{1}, \varepsilon_{2}$ to the system (3.1), along with the parameter $\eta$, converge to 0 and the shorter curve $\gamma$ converges to $\bar{\gamma}$.

We may look for solutions to (3.1) in the case $\alpha=1$. However, when $\alpha=1$, Eq. (3.3) for the case $\sigma=\varepsilon_{2}>0$ reads

$$
\eta^{4}(31-16 \eta)+8 \eta^{2} \sigma^{2}+45 \sigma^{4}+o\left(\sigma^{4}\right)=0
$$

This equation has no small solution $\sigma>0$ for small $\eta$.

On the other hand, when $\alpha=1$, Eq. (3.7) for the case $\sigma=\left|\varepsilon_{2}\right|$ with $\varepsilon_{2}<0$ reads

$$
\eta^{4}(31-16 \eta)-8 \eta^{2} \sigma^{2}+53 \sigma^{4}+o\left(\sigma^{4}\right)=0 .
$$

And also this equation has for small $\eta>0$ no small solution $\sigma>0$. 
Remark 3.2 The shorter curve $\gamma$ constructed above has a "curl" at the end-point $R$. When $\alpha>1$, this curl is oriented clockwise (i.e., $\varepsilon_{2}>0$ ). When $0<\alpha<1$, it is oriented counterclockwise (i.e., $\varepsilon_{2}<0$ ). This suggests that the geodesic joining $L$ to $R$ displays a similar behavior. One may wonder what is the behavior at the point $R$ of the length-minimizing curve joining $L$ to $R$ when $\alpha=1$.

Remark 3.3 The proof of Theorem 1.2 shows why the problem of extending Theorem 1.1 to situations where (1.6) fails is technically complicated. The presence of coefficients depending on nonhorizontal coordinates [as the coefficient $x_{3}^{2}$ in the vector field $X_{1}$ in (1.1)] makes the construction of horizontal competitors complicated.

\section{Nilpotent approximation}

Let $(M, \mathcal{D})$ be a 4-dimensional sub-Riemannian manifold satisfying $(\mathrm{H} 1)-(\mathrm{H} 3)$ at the point $p \in M$, where $\mathcal{D}=\operatorname{span}\left\{Y_{1}, Y_{2}\right\}$, and let $Y_{1}, \ldots, Y_{4}$ be linearly independent vector fields at the point $p$, as in (1.10). In this section, we construct the homogeneous nilpotent approximation of $(M, \mathcal{D})$ at $p$.

In a neighborhood of $p \in M$, we fix the exponential coordinates of the first type induced by the frame $Y_{1}, \ldots, Y_{4}$ starting from $p$. Then, we can identify $M$ with $\mathbb{R}^{4}, Y_{1}, \ldots, Y_{4}$ with vector fields on $\mathbb{R}^{4}$, and $p$ with $0 \in \mathbb{R}^{4}$. We have

$$
x=\exp \left(\sum_{i=1}^{4} x_{i} Y_{i}\right)(0), \quad x=\left(x_{1}, \ldots, x_{4}\right) \in \mathbb{R}^{4} .
$$

Here, the exponential mapping is defined by $\exp (Y)(0)=\gamma(1)$ where $\gamma$ is the solution of $\dot{\gamma}=Y(\gamma)$ and $\gamma(0)=0$.

We assign to the coordinate $x_{1}$ the weight $w_{1}=1$, to $x_{2}$ the weight $w_{2}=1$, to $x_{3}$ the weight $w_{3}=2$, and to $x_{4}$ the weight $w_{4}=5$. In fact, the length of $Y_{3}$ is len $\left(Y_{3}\right)=2$, and the length of $Y_{4}$ is $\operatorname{len}\left(Y_{4}\right)=5$. The natural dilations adapted to the frame $Y_{1}, \ldots, Y_{4}$ are

$$
\delta_{\lambda}(x)=\left(\lambda x_{1}, \lambda x_{2}, \lambda^{2} x_{3}, \lambda^{5} x_{4}\right), \quad x \in \mathbb{R}^{4}, \lambda>0 .
$$

Let $Y=Y_{\beta}$ be a commutator of $Y_{1}, \ldots, Y_{4}$, with the notation (1.8) for iterated commutators. Then, we have

$$
Y=\sum_{i=1}^{4} a_{i}(x) \frac{\partial}{\partial x_{i}}
$$

where $a_{i} \in C^{\infty}\left(\mathbb{R}^{4}\right), i=1, \ldots, 4$, are smooth functions that have the structure described in the following proposition.

Proposition 4.1 Assume that $(H 1)$ and $(H 2)$ hold. There are polynomials $p_{i}: \mathbb{R}^{4} \rightarrow \mathbb{R}$ and functions $r_{i}: \mathbb{R}^{4} \rightarrow \mathbb{R}, i=1, \ldots, 4$, such that:

i) $a_{i}(x)=p_{i}(x)+r_{i}(x), x \in \mathbb{R}^{4}$;

ii) $p_{i}\left(\delta_{\lambda}(x)\right)=\lambda^{w_{i}-\operatorname{len}(Y)} p_{i}(x)$, where len $(Y)$ is the length of $Y$;

iii) $\lim _{\lambda \rightarrow \infty} \lambda^{w_{i}-\operatorname{len}(Y)} r_{i}\left(\delta_{1 / \lambda}(x)\right)=0, x \in \mathbb{R}^{4}$.

Proposition 4.1 can be proved as in [4, on page 306]. We omit the details, here. 
Let $Y=Y_{\beta}$ be a vector field as in (4.2). For $\lambda>0$, we let

$$
Y^{\lambda}(x)=\sum_{i=1}^{4} \lambda^{w_{i}-\operatorname{len}(Y)} a_{i}\left(\delta_{1 / \lambda}(x)\right) \frac{\partial}{\partial x_{i}}, \quad x \in \mathbb{R}^{4} .
$$

The mapping $Y \mapsto Y^{\lambda}$ is bracket-preserving. Namely, for any multi-index $\beta$, and for $i=1,2$, we have

$$
\left[Y_{i}, Y_{\beta}\right]^{\lambda}=\left[Y_{i}^{\lambda}, Y_{\beta}^{\lambda}\right], \quad \lambda>0 .
$$

The vector fields $Y_{1}^{\lambda}, \ldots, Y_{4}^{\lambda}$ induce on $\mathbb{R}^{4}$ exponential coordinates of the first type starting from 0 :

$$
x=\exp \left(\sum_{i=1}^{4} x_{i} Y_{i}^{\lambda}\right)(0), \quad x \in \mathbb{R}^{4} .
$$

The proof of (4.5) relies upon the fact that if a curve $\gamma$ solves $\dot{\gamma}=\sum_{i=1}^{4} x_{i} Y_{i}(\gamma)$, then the curve $\gamma_{\lambda}=\delta_{\lambda}(\gamma)$ solves $\dot{\gamma}_{\lambda}=\sum_{i=1}^{4} \lambda^{w_{i}} x_{i} Y_{i}^{\lambda}\left(\gamma_{\lambda}\right)$.

By Proposition 4.1, for any $Y=Y_{\beta}$ as in (4.2), we can define the vector field $Y^{\infty}$ in $\mathbb{R}^{4}$

$$
Y^{\infty}(x)=\lim _{\lambda \rightarrow \infty} Y^{\lambda}(x)=\sum_{i=1}^{4} p_{i}(x) \frac{\partial}{\partial x_{i}}, \quad x \in \mathbb{R}^{4},
$$

where $p_{i}, i=1, \ldots, 4$, are polynomials such that $p_{i} \circ \delta_{\lambda}=\lambda^{w_{i}-\operatorname{len}(Y)} p_{i}$. In particular, if $w_{i}<\operatorname{len}(Y)$, then $p_{i}=0$. Passing to the limit as $\lambda \rightarrow \infty$ in (4.4), we see that also the mapping $Y \mapsto Y^{\infty}$ is bracket-preserving

$$
\left[Y_{i}, Y_{\beta}\right]^{\infty}=\left[Y_{i}^{\infty}, Y_{\beta}^{\infty}\right], \quad \lambda>0 .
$$

Moreover, passing to the limit as $\lambda \rightarrow \infty$ in (4.5), we see that $Y_{1}^{\infty}, \ldots, Y_{4}^{\infty}$ induce exponential coordinates of the first type:

$$
x=\exp \left(\sum_{i=1}^{4} x_{i} Y_{i}^{\infty}\right)(0), \quad x \in \mathbb{R}^{4} .
$$

Then, the vector fields $Y_{1}^{\infty}, \ldots, Y_{4}^{\infty}$ are

$$
\begin{aligned}
Y_{1}^{\infty} & =\frac{\partial}{\partial x_{1}}+q_{1} \frac{\partial}{\partial x_{3}}+p_{1} \frac{\partial}{\partial x_{4}}, \\
Y_{2}^{\infty} & =\frac{\partial}{\partial x_{2}}+q_{2} \frac{\partial}{\partial x_{3}}+p_{2} \frac{\partial}{\partial x_{4}}, \\
Y_{3}^{\infty} & =\frac{1}{4}\left[Y_{2}^{\infty}, Y_{1}^{\infty}\right]=\frac{\partial}{\partial x_{3}}+p_{3} \frac{\partial}{\partial x_{4}}, \\
Y_{4}^{\infty} & =\frac{1}{16}\left[Y_{1}^{\infty},\left[Y_{1}^{\infty},\left[Y_{2}^{\infty},\left[Y_{2}^{\infty}, Y_{1}^{\infty}\right]\right]\right]\right]=\frac{\partial}{\partial x_{4}} .
\end{aligned}
$$

Above, $q_{i}$ and $p_{i}$ are polynomials such that $q_{i} \circ \delta_{\lambda}=\lambda q_{i}$ and $p_{i} \circ \delta_{\lambda}=\lambda^{4} p_{i}$ for $i=1,2$ and $\lambda>0$. In particular, for $i=1,2$, we have

$$
q_{i}=a_{i 1} x_{1}+a_{i 2} x_{2} \text { and } p_{i}=p_{i 1}+x_{3} p_{i 2}+d_{i} x_{3}^{2},
$$


where $a_{i j}, d_{i} \in \mathbb{R}$ are constants, $p_{i 1}$ are homogeneous polynomials of degree 4 in the variables $x_{1}, x_{2}$, and $p_{i 2}$ are homogeneous polynomials of degree 2 in the variables $x_{1}, x_{2}$. By the relation $Y_{3}^{\infty}=\frac{1}{4}\left[Y_{2}^{\infty}, Y_{2}^{\infty}\right]$, we deduce that the polynomial $p_{3}$ is

$$
p_{3}=\frac{1}{4}\left\{\left(\frac{\partial}{\partial x_{2}}+q_{2} \frac{\partial}{\partial x_{3}}\right) p_{1}-\left(\frac{\partial}{\partial x_{1}}+q_{1} \frac{\partial}{\partial x_{3}}\right) p_{2}\right\} .
$$

If $Y_{1}, \ldots, Y_{4}$ satisfy (1.12), then the vector fields $Y_{1}^{\infty}, \ldots, Y_{4}^{\infty}$ satisfy, for all $\beta \in \mathcal{I}_{5}$ with $\beta=(*, *, *, 2,1)$,

$$
\begin{aligned}
& Y_{\beta}^{\infty}(0)=16 Y_{4}^{\infty}(0) \text { for } \beta=(1,2,1,2,1) \text { or } \beta=(1,1,2,2,1), \\
& Y_{\beta}^{\infty}(0)=-32 Y_{4}^{\infty}(0) \text { for } \beta=(2,1,1,2,1), \text { and } \\
& Y_{\beta}^{\infty}(0)=0 \text { otherwise. }
\end{aligned}
$$

We claim that

$$
Y_{1}^{\infty}=X_{1} \text { and } Y_{2}^{\infty}=X_{2},
$$

where $X_{1}$ and $X_{2}$ are the vector fields (1.1).

For any $x \in \mathbb{R}^{4}$, let $\gamma:[0,1] \rightarrow \mathbb{R}^{4}$ be the curve such that $\dot{\gamma}=\exp \left(\sum_{i=1}^{4} x_{i} Y_{i}^{\infty}(\gamma)\right)(0)$ and $\gamma(0)=0$. Condition (4.7) implies that $\gamma(t)=t x$ for all $t \in[0,1]$. Differentiating this identity at $t=1$, we obtain

$$
\sum_{i=1}^{4} x_{i} \frac{\partial}{\partial x_{i}}=\sum_{i=1}^{4} x_{i} Y_{i}^{\infty}(x), \quad x \in \mathbb{R}^{4} .
$$

The equation for the coordinate $i=3$ in (4.12) is $x_{1} q_{1}+x_{2} q_{2}=0$, i.e.,

$$
\sum_{i, j=1}^{2} a_{i j} x_{i} x_{j}=0 \text {. }
$$

This equation along with the relation $\frac{1}{4}\left[Y_{2}^{\infty}, Y_{1}^{\infty}\right](0)=\partial / \partial x_{3}$ gives

$$
q_{1}=2 x_{1} \text { and } q_{2}=-2 x_{1} .
$$

The equation for the coordinate $i=4$ in (4.12) is $x_{1} p_{1}+x_{2} p_{2}+x_{3} p_{3}=0$. By (4.13) and (4.9), this equation reads

$$
x_{1} p_{1}+x_{2} p_{2}+\frac{x_{3}}{4}\left(\partial_{2} p_{1}-2 x_{1} \partial_{3} p_{1}-\partial_{1} p_{2}-2 x_{2} \partial_{3} p_{2}\right)=0
$$

or equivalently,

$$
x_{1} p_{11}+x_{2} p_{21}+\frac{x_{3}}{4}\left(2 x_{1} p_{12}+2 x_{2} p_{22}+\partial_{2} p_{11}-\partial_{1} p_{21}\right)+\frac{x_{3}^{2}}{4}\left(\partial_{2} p_{12}-\partial_{1} p_{22}\right)=0 .
$$

Setting to 0 the coefficients of the powers of $x_{3}$ in (4.14), we obtain the following equations:

$$
\begin{aligned}
& x_{1} p_{11}+x_{2} p_{21}=0, \\
& 2 x_{1} p_{12}+2 x_{2} p_{22}+\partial_{2} p_{11}-\partial_{1} p_{21}=0, \\
& \partial_{2} p_{12}-\partial_{1} p_{22}=0 .
\end{aligned}
$$

Equation (4.15) implies that

$$
p_{11}=x_{2} p \text { and } p_{21}=-x_{1} p,
$$


where

$p$ is a homogeneous polynomial of degree 3 in the variables $x_{1}, x_{2}$.

Now, Eq. (4.16) reads

$$
5 p+2 x_{1} p_{12}+2 x_{2} p_{22}=0 .
$$

The vector fields $Y_{1}^{\infty}$ and $Y_{2}^{\infty}$ are

$$
Y_{1}^{\infty}=X_{1}+w_{1} \frac{\partial}{\partial x_{4}} \text { and } Y_{2}^{\infty}=X_{2}+w_{2} \frac{\partial}{\partial x_{4}},
$$

where $X_{1}, X_{2}$ are the vector fields (1.1), and

$$
\begin{aligned}
& w_{1}=x_{2} p+x_{3} p_{12}+\left(d_{1}-1\right) x_{3}^{2}, \\
& w_{2}=-x_{1} p+x_{3} p_{22}+d_{2} x_{3}^{2} .
\end{aligned}
$$

We claim that $w_{1}=w_{2}=0$. Once this claim is proved, the main claim (4.11) will follow.

We compute commutators of length 5 of $Y_{1}^{\infty}$ and $Y_{2}^{\infty}$, as functions of $X_{1}, X_{1}, w_{1}$, and $w_{2}$. First of all, we have

$$
\left[Y_{2}^{\infty}, Y_{1}^{\infty}\right]=\left[X_{2}, X_{1}\right]+\left(X_{2} w_{1}-X_{1} w_{2}\right) \frac{\partial}{\partial x_{4}} .
$$

Then, for any $i, j, k=1,2$, we have

$$
\begin{aligned}
{\left[Y_{i}^{\infty},\left[Y_{j}^{\infty},\left[Y_{k}^{\infty},\left[Y_{2}^{\infty}, Y_{1}^{\infty}\right]\right]\right]\right]=} & {\left[X_{i},\left[X_{j},\left[X_{k},\left[X_{2}, X_{1}\right]\right]\right]\right] } \\
& +\left(X_{i} X_{j} X_{k} X_{2} w_{1}-X_{i} X_{j} X_{k} X_{1} w_{2}-4 X_{i} X_{j} \partial_{3} w_{k}\right) \frac{\partial}{\partial x_{4}} .
\end{aligned}
$$

By (1.9) and (4.10), commutators (of length 5) of $Y_{1}^{\infty}$ and $Y_{2}^{\infty}$, and commutators of $X_{1}$ and $X_{2}$ satisfy the same relations at the point $x=0$. From (4.22), we deduce that we have for all $i, j, k=1,2$

$$
X_{i} X_{j} X_{k} X_{2} w_{1}-X_{i} X_{j} X_{k} X_{1} w_{2}-4 X_{i} X_{j} \partial_{3} w_{k}=0, \quad \text { at } x=0 .
$$

Notice that $X_{i} X_{j} X_{k} X_{2} w_{1}-X_{i} X_{j} X_{k} X_{1} w_{2}-4 X_{i} X_{j} \partial_{3} w_{k}$ is a homogeneous polynomial of degree 0 for the dilations $\left(x_{1}, x_{2}, x_{3}\right) \mapsto\left(\lambda x_{1}, \lambda x_{2}, \lambda^{2} x_{3}\right), \lambda>0$. Thus, Eq. (4.23) holds for all $x \in \mathbb{R}^{4}$. By integration, we deduce that $X_{j} X_{k} X_{2} w_{1}-X_{j} X_{k} X_{1} w_{2}-4 X_{j} \partial_{3} w_{k}$ is constant in $\mathbb{R}^{3}$. For $X_{j} X_{k} X_{2} w_{1}-X_{j} X_{k} X_{1} w_{2}-4 X_{j} \partial_{3} w_{k}$ is a homogeneous polynomial of degree 1 , we deduce that it is identically zero. Repeating the same argument, we conclude that for $k=1,2$

$$
X_{k} X_{2} w_{1}-X_{k} X_{1} w_{2}-4 \partial_{3} w_{k}=0 \text { in } \mathbb{R}^{3} .
$$

In order to analyze Eq. (4.24), we preliminarily compute

$$
\begin{aligned}
& X_{2} w_{1}=p+x_{2} \partial_{2} p+x_{3} \partial_{2} p_{12}-2 x_{1} p_{12}-4\left(d_{1}-1\right) x_{1} x_{3}, \\
& X_{1} w_{2}=-p-x_{1} \partial_{1} p+x_{3} \partial_{1} p_{22}+2 x_{2} p_{22}+4 d_{2} x_{2} x_{3} .
\end{aligned}
$$

When $k=1$, Eq. (4.24) reads

$$
\begin{aligned}
& 3 \partial_{1} p+x_{1} \partial_{1} \partial_{1} p+x_{2} \partial_{1} \partial_{2} p-6 p_{12}-2 x_{1} \partial_{1} p_{12}-2 x_{2} \partial_{1} p_{22}+2 x_{2}\left(\partial_{2} p_{12}-\partial_{1} p_{22}\right)+ \\
& -8\left(d_{1}-1\right) x_{1} x_{2}-8 d_{2} x_{2}^{2}+x_{3}\left(\partial_{1} \partial_{2} p_{12}-\partial_{1} \partial_{1} p_{22}-12\left(d_{1}-1\right)\right)=0 .
\end{aligned}
$$


As $\partial_{1} p$ is a homogeneous polynomial of degree 2, we have $x_{1} \partial_{1} \partial_{1} p+x_{2} \partial_{2} \partial_{1} p=2 \partial_{1} p$. Also using the fact that $p_{12}$ is a homogeneous polynomial of degree 2 , and using identity (4.17), the previous equation reads

$$
5 \partial_{1} p-10 p_{12}-8\left(d_{1}-1\right) x_{1} x_{2}-8 d_{2} x_{2}^{2}-12\left(d_{1}-1\right) x_{3}=0 .
$$

This implies

$$
d_{1}-1=0 \text { and } \partial_{1} p-2 p_{12}-\frac{8}{5} d_{2} x_{2}^{2}=0 .
$$

When $k=2$, Eq. (4.24) reads

$$
\begin{aligned}
& 3 \partial_{2} p+x_{1} \partial_{2} \partial_{1} p+x_{2} \partial_{2} \partial_{2} p-6 p_{22}-2 x_{1} \partial_{2} p_{12}-2 x_{2} \partial_{2} p_{22}+2 x_{1}\left(\partial_{1} p_{22}-\partial_{2} p_{12}\right) \\
& \quad+8\left(d_{1}-1\right) x_{1}^{2}+8 d_{2} x_{1} x_{2}+x_{3}\left(\partial_{2} \partial_{2} p_{12}-\partial_{2} \partial_{1} p_{22}-12 d_{2}\right)=0 .
\end{aligned}
$$

Using the fact that $\partial_{2} p$ is homogeneous of degree 2, identity (4.17), the fact that $p_{22}$ is homogeneous of degree 2 , and $d_{1}=1$, the previous equation reads

$$
5 \partial_{2} p-10 p_{22}+8 d_{2} x_{1} x_{2}-12 d_{2} x_{3}=0 .
$$

This implies $d_{2}=0$ and $\partial_{2} p-2 p_{22}=0$. We multiply the latter equation by $x_{2}$, we multiply by $x_{1}$ the second equation in (4.25) (with $d_{2}=0$ ), we sum the two resulting equations, and we obtain $x_{1} \partial_{1} p+x_{2} \partial_{2} p-2 x_{1} p_{12}-2 x_{2} p_{22}=0$. Using the fact that $p$ is homogeneous of degree 3 , we finally obtain

$$
3 p-2 x_{1} p_{12}-2 x_{2} p_{22}=0 .
$$

From (4.26) and (4.20), we deduce that $p=0$, and thus, $p_{12}=p_{22}=0$. We conclude that $w_{1}=w_{2}=0$, and this proves the main claim (4.11).

\section{Proof of Theorem 1.3}

In this section, we prove Theorem 1.3. As in Sect. 4, we assume that $M=\mathbb{R}^{4}, p=0, \mathcal{D}=$ $\operatorname{span}\left\{Y_{1}, Y_{2}\right\}$ and that the vector fields $Y_{1}, \ldots, Y_{4}$ satisfy (H1)-(H3) and induce exponential coordinates of the first type in $\mathbb{R}^{4}$, as in (4.1). On $\mathcal{D}$, we fix a metric $g$ satisfying (1.13).

For $\lambda>0$, let $Y_{1}^{\lambda}$ and $Y_{2}^{\lambda}$ be the vector fields in (4.3), and let $\mathcal{D}^{\lambda}=\operatorname{span}\left\{Y_{1}^{\lambda}, Y_{2}^{\lambda}\right\}$. Let $g^{\lambda}$ be the metric on $\mathcal{D}^{\lambda}$ defined by

$$
g_{x}^{\lambda}\left(Y_{i}^{\lambda}, Y_{j}^{\lambda}\right)=g_{\delta_{1 / \lambda}(x)}\left(Y_{i}, Y_{j}\right), \quad x \in \mathbb{R}^{4}, \quad i, j=1,2 .
$$

When $\lambda=\infty$, we let $\mathcal{D}^{\infty}=\operatorname{span}\left\{Y_{1}^{\infty}, Y_{2}^{\infty}\right\}$, where, by the discussion of Sect. 4 , we have $Y_{1}^{\infty}=X_{1}$ and $Y_{2}^{\infty}=X_{2}$. On $\mathcal{D}^{\infty}$, we define the metric $g^{\infty}$

$$
g_{x}^{\infty}\left(Y_{i}^{\infty}, Y_{j}^{\infty}\right)=\lim _{\lambda \rightarrow \infty} g_{x}^{\lambda}\left(Y_{i}^{\lambda}, Y_{j}^{\lambda}\right)=\lim _{\lambda \rightarrow \infty} g_{\delta_{1 / \lambda}(x)}\left(Y_{i}, Y_{j}\right)=g_{0}\left(Y_{i}, Y_{j}\right), \quad x \in \mathbb{R}^{4} .
$$

Remark 5.1 From (1.13), it follows that

$$
g_{x}^{\infty}\left(Y_{1}^{\infty}, Y_{2}^{\infty}\right)=g_{0}\left(Y_{1}, Y_{2}\right)=0, \quad x \in \mathbb{R}^{4} .
$$

Without loss of generality, we also assume that $g_{x}^{\infty}\left(Y_{i}^{\infty}, Y_{i}^{\infty}\right)=1$ for all $x \in \mathbb{R}^{4}$ and $i=1,2$. In other words, the metric $g^{\infty}$ makes the vector fields $Y_{1}$ and $Y_{2}$ orthonormal. It follows that linear mappings $T: \mathbb{R}^{4} \rightarrow \mathbb{R}^{4}$ of the form

$$
T(x)=\left(U\left(x_{1}, x_{2}\right), \operatorname{det}(U) x_{3}, x_{4}\right), \quad x=\left(x_{1}, \ldots, x_{4}\right) \in \mathbb{R}^{4},
$$


where $U \in O(2)$ is an orthogonal mapping in $\mathbb{R}^{2}$, are isometries of $\left(\mathbb{R}^{4}, \mathcal{D}^{\infty}, g^{\infty}\right)$.

Let $\gamma:[-1,1] \rightarrow M=\mathbb{R}^{4}$ be a $\mathcal{D}$-horizontal curve such that $\gamma(0)=0$ and

$$
\dot{\gamma}_{L}(0) \neq \dot{\gamma}_{R}(0)
$$

where $\dot{\gamma}_{L}(0) \neq 0$ and $\dot{\gamma}_{R}(0) \neq 0$ are the left and right derivatives of $\gamma$ at $t=0$.

For $\lambda>0$, the curves $\gamma^{\lambda}(t)=\delta_{\lambda} \gamma(t / \lambda), t \in[-\lambda, \lambda]$, are $\mathcal{D}^{\lambda}$-horizontal. Moreover, the limit curve $\gamma^{\infty}:(-\infty, \infty) \rightarrow \mathbb{R}^{4}$

$$
\gamma^{\infty}(t)=\left\{\begin{array}{l}
\lim _{\lambda \rightarrow \infty} \gamma^{\lambda}(t)=\dot{\gamma}_{R}(0) t t \geq 0, \\
\lim _{\lambda \rightarrow \infty} \gamma^{\lambda}(t)=\dot{\gamma}_{L}(0) t t<0,
\end{array}\right.
$$

is $\mathcal{D}^{\infty}$-horizontal. Here, the vectors $\dot{\gamma}_{L}(0)$ and $\dot{\gamma}_{R}(0)$ are identified with vectors of $\mathbb{R}^{4}$ and have the form $(*, *, 0,0)$. The curve $\gamma^{\infty}$ lies, therefore, in the $x_{1} x_{2}$-plane.

Proposition 5.1 Assume that the curve $\gamma$ is length minimizing in $\left(\mathbb{R}^{4}, \mathcal{D}, g\right)$. Then, the curve $\gamma^{\infty}$ is length minimizing in $\left(\mathbb{R}^{4}, \mathcal{D}^{\infty}, g^{\infty}\right)$.

Proposition 5.1 is proved in [2], Proposition 2.4.

We conclude the proof of Theorem 1.3. Assume by contradiction that there exists a curve $\gamma$ as in Theorem 1.3 that is length minimizing in $\left(\mathbb{R}^{4}, \mathcal{D}, g\right)$. By Proposition 5.1, the curve $\gamma^{\infty}$ in (5.2) is length minimizing in $\left(\mathbb{R}^{4}, \mathcal{D}^{\infty}, g^{\infty}\right)$. By assumption, we have $g_{0}\left(\dot{\gamma}_{L}(0), \dot{\gamma}_{R}(0)\right) \neq 0$. It follows that

$$
g_{0}^{\infty}\left(\dot{\gamma}_{L}^{\infty}(0), \dot{\gamma}_{R}^{\infty}(0)\right)=\lim _{\lambda \rightarrow \infty} g_{0}^{\lambda}\left(\dot{\gamma}_{L}^{\lambda}(0), \dot{\gamma}_{R}^{\lambda}(0)\right)=g_{0}\left(\dot{\gamma}_{L}(0), \dot{\gamma}_{R}(0)\right) \neq 0 .
$$

By Remark 5.1 and (5.1), we can assume that $\gamma^{\infty}$ has the form (1.5) with $\alpha>0$ (up to re-parameterization), and by (5.3), we have $\alpha \neq 1$. By Theorem 1.2, the curve $\gamma^{\infty}$ is not length minimizing. This is a contradiction.

\section{References}

1. Le Donne, E., Leonardi, G.P., Monti, R., Vittone, D.: Extremal curves in nilpotent Lie groups. Geom. Funct. Anal. (2013, to appear)

2. Leonardi, G.P., Monti, R.: End-point equations and regularity of sub-Riemannian geodesics. Geom. Funct. Anal. 18, 552-582 (2008)

3. Liu, W., Sussmann, H.: Shortest paths for sub-Riemannian metrics on rank-two distributions. Mem. Am. Math. Soc. 118, $\mathrm{x}+104(1995)$

4. Margulis, G.A., Mostow, G.D.: Some remarks on the definition of tangent cones in a Carnot-Carathèodory space. J. Anal. Math. 80, 299-317 (2000)

5. Montgomery, R.: Abnormal minimizers. SIAM J. Control Optim. 32, 1605-1620 (1994)

6. Monti, R.: Regularity results for sub-Riemannian geodesics. Calc. Var. (2013, to appear) 